\begin{tabular}{|c|l|}
\hline Title & A utoimmune retinopathy associated with colonic adenoma \\
\hline Author(s) & Saito, Wataru; Kase, Satoru; Ohguro, Hiroshi; Ishida, Susumu \\
\hline Citation & $\begin{array}{l}\text { Graefe's A rchive for Clinical and Experimental Ophthalmology, 251(5), 1447-1449 } \\
\text { https://doi.org/L0.1007/300417-012-2131-4 }\end{array}$ \\
\hline Issue Date & 2013-05 \\
\hline Doc URL & http://hdl.handle.net/2115/55279 \\
\hline Rights & The original publication is available at www.springerlink.com \\
\hline Type & article (author version) \\
\hline File Information & polyp_CARmanuscript.pdf \\
\hline
\end{tabular}

Instructions for use 


\section{Autoimmune retinopathy associated with colonic adenoma}

Wataru Saito, $\mathrm{MD}, \mathrm{PhD}^{1)}$, Satoru Kase, $\mathrm{MD}, \mathrm{PhD}^{1)}$, Hiroshi Ohguro, MD, $\mathrm{PhD}^{2)}$, and Susumu Ishida, $\mathrm{MD}, \mathrm{PhD}^{1)}$

1) Department of Ophthalmology, Hokkaido University Graduate School Medicine, Sapporo, Japan

2) Department of Ophthalmology, Sapporo Medical University School of Medicine, Sapporo, Japan

Correspondence to: Wataru Saito, Department of Ophthalmology, Hokkaido University Graduate School of Medicine, Nishi 7, Kita 15, Kita-ku, Sapporo 060-8638, Japan

Phone: $+81-11-706-5944$

Fax: $+81-11-706-5948$

E-mail: wsaito@med.hokudai.ac.jp

Conflict of interest: None 


\section{Dear Editor,}

Autoimmune retinopathy (AIR), retinal degeneration caused by antiretinal antibodies, is divided into 3 subtypes: cancer-associated retinopathy (CAR), melanoma-associated retinopathy, and non-paraneoplastic AIR (npAIR) [1]. Antiretinal antibodies in CAR are produced by cross-reactivity with aberrantly expressed tumour pathogens. Various malignancies cause CAR $[1,2]$. However, very few cases of AIR associated with benign tumours have been reported [3]. We report a case of antirecoverin antibody-positive AIR associated with colonic adenoma.

A 73-year-old woman presented with night blindness and central visual acuity loss of 5-year duration in the left eye. The patient had no medical or family history. The visual acuities were 0.9 OD and 0.2 OS. Slit lamp examination showed normal appearance. Funduscopic examination showed retinal pigment epithelial atrophy without pigmentation at the midperipheral area and cystoid macular oedema (CMO) (Fig. 1a). Fluorescein angiography showed an initial window defect corresponding to retinal degeneration and late leakage from retinal capillaries (Fig. 1b). Goldmann perimetry showed ring scotomata. Optical coherence 
tomography showed $\mathrm{CMO}$ and diffuse photoreceptor inner-segment outer-segment junction loss at the macula. Single bright-flash electroretinography showed bilateral reduced a-waves and normal b-waves. Western blot analysis did not detect retinal antibodies. The patient received sub-Tenon or intravitreal injections of triamcinolone acetonid (TA) for the CMO in the left eye. Central retinal thickness (CMT) decreased after each injection but increased in the intervening time.

One year after the first visit, Western blot analysis of the patient's serum detected antibody for 23-kDa recoverin (Fig. 2a), but systemic screening detected no malignancy. Two years after the first visit, visual acuities decreased to $0.5 \mathrm{OD}$ and $0.3 \mathrm{OS}$. A second systemic screening detected no malignancy; however, she underwent endoscopic mucosal resection for a colonic polyp. Three months later, visual acuity remained unchanged, but CMT decreased to $475 \mu \mathrm{m}$ OD and $270 \mu \mathrm{m}$ OS, compared to $533 \mu \mathrm{m}$ OD and $427 \mu \mathrm{m}$ OS before surgery, despite the lack of treatment (Fig. 1c, d). The histological features of the polyp were consistent with those of tubular adenoma with mild atypia (Fig. 2b, arrows). Immunoreactivity for recoverin was clearly detected in the cytoplasm of 
adenoma cells (Fig. 2c-e: green, arrows) but not in the normal mucosa. Therefore, the patient was diagnosed with AIR associated with colonic adenoma. She received oral prednisolone therapy $(20 \mathrm{mg} / \mathrm{d})$ and a sub-Tenon TA injection ( $40 \mathrm{mg}$ ) in the right eye; visual acuity was $0.5 \mathrm{OD}$ and $0.5 \mathrm{OS}$, with further CMT decrease $(259 \mu \mathrm{m}$ OD and $211 \mu \mathrm{m}$ OS $)$.

In the present case with AIR, CMT spontaneously reduced after colonic polyp resection, suggesting that tumour resection aided in treating paraneoplastic retinopathy. Additionally, the immunohistochemistry results suggest that the colonic adenoma cells aberrantly expressed recoverin, which caused the AIR. To our knowledge, this is the first report of AIR probably caused by benign colonic tubular adenoma.

We have previously reported a case of CAR caused by a small bronchioloalveolar carcinoma [4], with slow progressive visual deterioration, similar to this case. On the basis of the clinical course and immunohistochemical findings, we inferred that preneoplastic tumours express the pathogen responsible for AIR. The results of previous studies and this study confirm this speculation. Therefore, ophthalmologists should perform repeated systemic screening and identify and resect malignant and 
benign tumours, and even small polyps, in patients with presumed AIR. In conclusion, benign tumours, including colonic adenomas, can also cause AIR. Benign tumors might also correlate with npAIR pathogenesis because some of these patients have systemic benign tumours [5]. Therefore, we advocate that benign tumour-associated AIR should be considered as a new AIR subtype. 
Acknowledgements

None 


\section{References}

1. Heckenlively JR, Ferreyra HA (2008) Autoimmune retinopathy: a review and summary. Semin Immunopathol 30: 127-134

2. Chan JW (2003) Paraneoplastic retinopathies and optic neuropathies. Surv Ophthalmol 48: 12-38

3. Yamada G, Ohguro H, Aketa K, Itoh T, Shijubo N, Takahashi H, Fujiwara O, Satoh M, Ohtsuka K, Abe S (2003) Paraneoplastic retinopathy associated with invasive thymoma. Hum Pathol 34: $717-719$

4. Saito W, Kase S, Ohguro H, Furudate N, Ohno S (2007) Slowly progressive cancer-associated retinopathy. Arch Ophthalmol 125: $1431-1433$

5. Heckenlively JR, Fawzi AA, Oversier J, Jordan BL, Aptsiauri N (2000) Autoimmune retinopathy: patients with antirecoverin immunoreactivity and panretinal degeneration. Arch Ophthalmol 118: 1525-1533 


\section{Figure legends}

Fig. 1 Photographs of the left eye of a 73-year-old woman with antirecoverin antibody-positive autoimmune retinopathy.

a Fundus photograph showing cystoid macular oedema and retinal pigment epithelial atrophy in the midperipheral area on initial visit. b Fluorescein angiography (116 s after dye injection) showing a window defect corresponding to retinal degeneration and dye leakages from retinal capillaries. c, d Optical coherence tomography images obtained before and after resection of the colonic polyp. Central retinal thickness decreased to $270 \mu \mathrm{m}$ (d) without any treatment, compared to $427 \mu \mathrm{m}$ (c) before surgery

Fig. 2 Images for Western blot analysis performed using bovine retinal soluble fractions of the patient's serum (a), histopathological analysis (b), DAPI nuclear staining (c, e: blue), and recoverin immunoreactivity (d, e: green) performed on the resected colonic polyp.

a Antibody to a soluble $23-\mathrm{kDa}$ (recoverin) protein was probed with the patient's serum (lane S, 1:400 dilution). Lane R shows human recombinant recoverin. b Histologically, the colonic polyp is tubular adenoma with mild 
atypia (arrows). The normal mucosa is admixed (arrowheads) within adenoma tissue. The bar indicates $50 \mu \mathrm{m}$. c-e Immunoreactivity for recoverin is clearly detected in the cytoplasm of adenoma cells (green, arrows) but not in the normal mucosa (green, arrowheads) 
Figure1
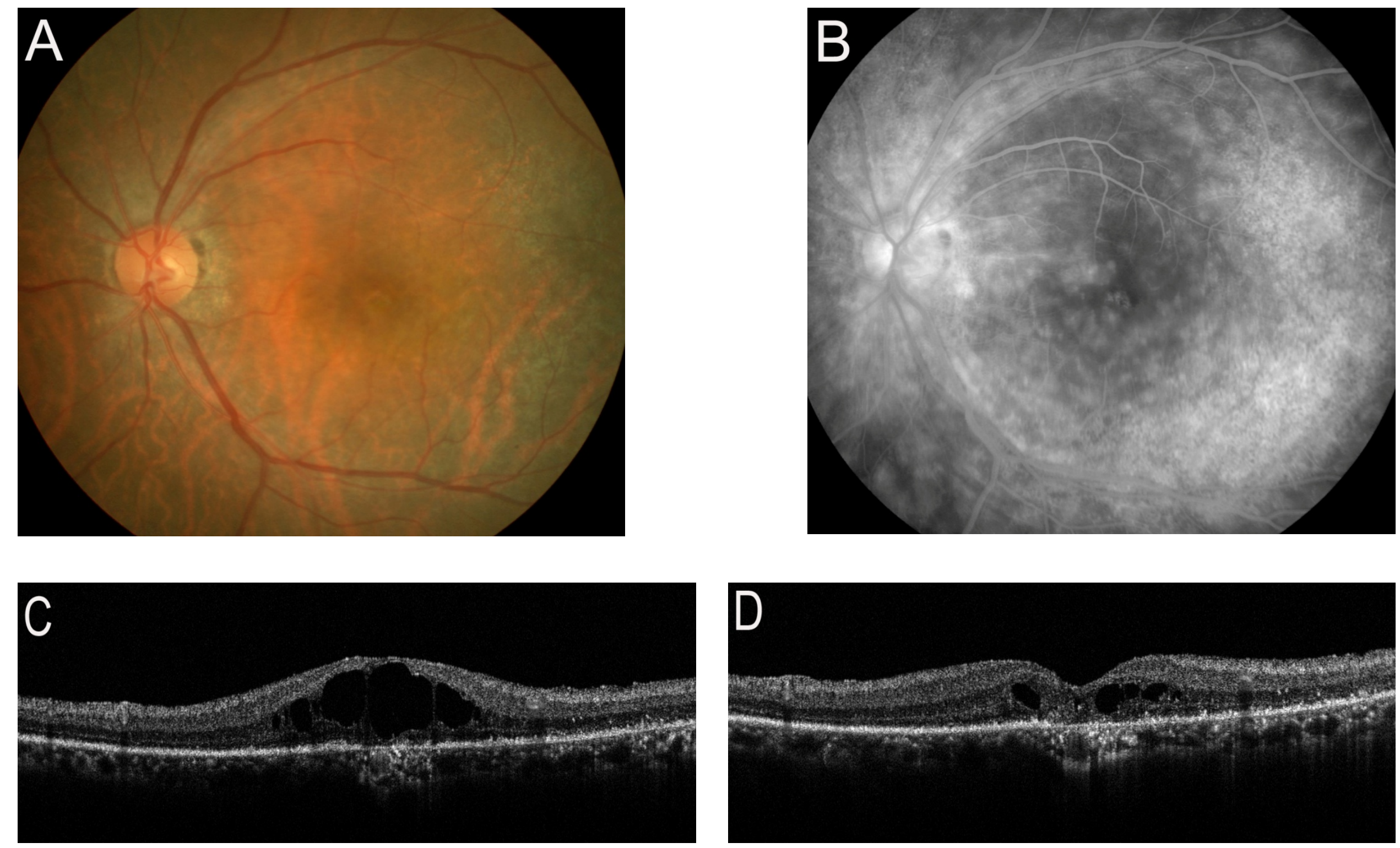


\section{Figure2}

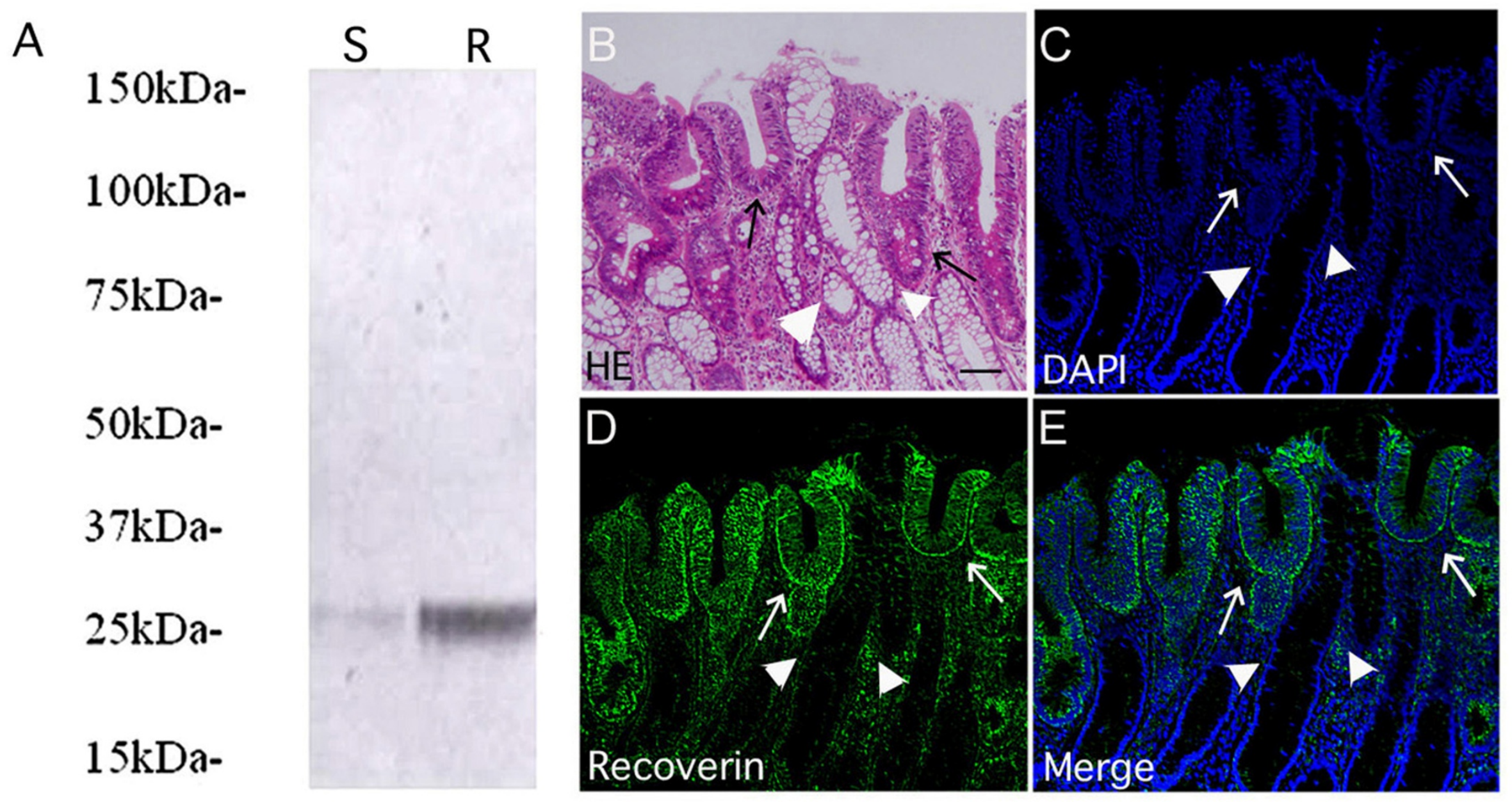

